\title{
Editorial: Smart Grid Advancements - Presentations From SPIES 2020
}

\author{
Ahmed Al-Durra * \\ Advanced Power and Energy Center, EECS Department, Khalifa University, Abu Dhabi, United Arab Emirates
}

Keywords: smart power, internet energy systems, smart grid informatics, advancement in demand response, power analyzer, space power modules

Editorial on the Research Topic

Smart Grid Advancements - Presentations From SPIES 2020

DRIVEN by the urgent need to realize a cleaner and more efficient, reliable, resilient, and responsive power grid, the energy sector is moving into the era of Smart Grids (SGs). The main concept in a typical SG is the evolution of a centralized, partially-automated, producer-controlled network to a decentralized one that minimizes environmentally adverse impacts; maximizes grid reliability and performance; and enables realtime interactions between customers, network operators, and power producers. This is a conceptual system in which all smart features are implemented in order to increase the efficient, and achieve more reliable and sustainable electrical distribution system. Distributed Generation, Nano-grids, Micro-grids with and without storage are now emerging as common features of today's complex power system. With the blessing of modern computer, communication, and information technologies, the traditional power system is going to be connected with Smart-grid hub.

Advancement of the technologies and devices are the key elements to enhance the utilization of energy in an economical and environmental friendly way. It can bring a considerable change in the conventional grid and consumer behavior towards utilization of energy by improving reliability, efficiency and quality of power delivery. Governmental policies are needed to facilitate smart grid implementation. This special issue will focus on the need of modernization of the conventional grid and how researchers are implementing modern grid concepts for electric power generation, transmission, and distribution networks in different aspects such as Asset Performance Management, Advanced Distribution Management Systems, and the convergence of Information Technology and Operations Technology within an electric utility to enable effective Enterprise Data Management are sources of encouragement in Smart grid technology.

This Special Issue of Frontiers in Energy Research presents expansions of selected papers from the 2020 International Conference on Smart Power and Internet Energy Systems (SPIES 2020). Authors of selected high-quality papers presented in the conference were invited to submit the extended versions of their papers to this Special Issue in Frontiers in Energy Research. This Special Issue aims to present novel research on smart grid advancements; Topics of interest include the following:

- Asset Performance Management.

- Energy-Efficiency Technologies

- Renewable Energy Sources and Systems

- Advanced Distribution Management Systems advanced real-time and Software applications.

- Power System Generation, Transmission, and Distribution

- Power Electronics Applications in Power System

- Big data, analytics, enterprise data management.

- Smart Grid Energy Informatics, Management, and Optimization

- Technological Advancements Needed for a Smarter Grid

- Smart Grid standards and interoperability 
Six papers were accepted and are presented in this Issue.

\section{TOPIC A: SPACE HIGH-VOLTAGE POWER MODULE}

Zhao et al. propose a high-voltage power supply module design for space welding applications, which outputs $1 \mathrm{kV}$ and $200 \mathrm{~W}$ when the input is $100 \mathrm{~V}$. It is quite difficult for space power supplies to directly achieve high-voltage output from the bus, because of the harshness of the space environment and the performance limitations of existing aerospace-grade electronic components. This paper also improves the efficiency of the high-voltage converter with a phase-shifted full-bridge series resonant circuit, then simulates the optimized power module and the electric field distribution of the high-voltage circuit board.

\section{TOPIC B: PSO SUPPORTED ENSEMBLE ALGORITHM FOR BAD DATA DETECTION AGAINST INTELLIGENT HACKING ALGORITHM}

Yavuz et al. propose a novel machine learning (ML) based detection algorithm that combines the five most popular ML algorithms with Particle Swarm Optimizer (PSO) using an intelligent hacking algorithm that is specially developed to measure the effectiveness of this study. The hacking algorithm provides three different types of injections: random, continuous random, and slow injections by adaptive manner. This would make detection harder. Results shows that recall values with the proposed algorithm for each different type of attack have been increased.

\section{TOPIC C: BESS AS A UPS TO POWER SYSTEMS WITH HIGH SOLAR PENETRATION}

Aluthge et al. analyzed the dynamic stability issues of the Sri Lankan power system as the solar power penetration is increased resulting in severe machine tripping. Remedial action needed to be taken to increase the power quality of the power system, namely, BESS and supercapacitors are presented. A comparison of the inertia of the current system and the system when conventional power replaces solar power is also analyzed. The article addresses on how fast-active power injection could be used as an uninterrupted power supply to the selected feeder. Further, an approach is also taken to establish the required reactive power to the feeder as well.

\section{TOPIC D: PROGRESS IN DEMAND RESPONSE AND IT'S INDUSTRIAL APPLICATIONS}

Siddiquee et al. provide a comprehensive review of demand response and it's industrial application by addressing: 1) Current research status, 2) Current stages of demand response applications in industries, and 3) Barriers in the deployment of DR programs. This study shows that there is significant research progress in recent years in the field of DR. It also shows potential applications of DR programs in industries. However, the study found several technical, policy, and financial barriers still exist, limiting the widespread adoption of DR. Thus, this paper offers recommendations on technical, policy, and financial measures needed to over-come the barriers and help facilitate the utilization of demand response potential, especially in industries.

\section{TOPIC E: A HIGH-PRECISION AND WIDEBAND FUNDAMENTAL FREQUENCY MEASUREMENT METHOD FOR SYNCHRONOUS SAMPLING USED IN THE
POWER ANALYZER}

Wang et al. propose a new fundamental frequency measurement architecture used in synchronous sampling to address the difficulty of obtaining accurate fundamental frequency measurement due to the harmonics and noise in the power signal. This architecture consists of a small-point fast Fourier transform module, spectrum refinement algorithm, and a multimodal optimization method to calculate the accurate fundamental frequency under large harmonic conditions. In the practical hardware platform results, this architecture has a large fundamental frequency measurement range from 20 to $200 \mathrm{kHz}$ with a relative error which is $<0.004 \%$. The wideband fundamental frequency measurement structure proposed in this article achieves high measurement accuracy.

\section{TOPIC F: INVESTIGATION OF OPTIMAL PV ALLOCATION TO MINIMIZE SYSTEM LOSSES AND IMPROVE VOLTAGE STABILITY FOR DISTRIBUTION AND TRANSMISSION NETWORKS USING MATLAB AND DIGSILENT}

Rasheed et al. propose methods to optimize the size, location, and power factor of PV-DG units. The simulations are performed on the IEEE 33 bus radial distribution network and IEEE 14 bus transmission network. The methodologies developed in this article are divided into two sections. The first section aims to optimize the PV-DG size and location. A multi-objective function is developed by using system losses and a voltage deviation index. Genetic algorithm (GA) is used to optimize the multi-objective function. Next, analytical processes are developed for verification. The second section aims to further enhance PV-DG by optimizing the power factor of PV-DG. A mathematical analytical method was developed, and it was found to be sufficient to optimize the power factor of the PV-DG unit. The results obtained show that voltage stability indices help minimize the computation time by determining the optimal locations for DG placement in both networks. In addition, the GA method attained faster convergence than the analytical method and 
hence is the best optimal sizing for both test systems with minimum computation time. Additionally, the optimization of the power factor for both test systems has demonstrated further improvement in the voltage profile and loss minimization.

\section{SUMMARY/CONCLUSION}

All of the papers select for this Special Issue show a timely and interesting topic in smart grid advancements. The Guest Editorial Board appreciates all contributions of the authors to the Special Issue, Selected Papers from the 2020 second International Conference on Smart Power and Internet Energy Systems (SPIES 2020). Moreover, they express their gratitude to the esteemed reviewers who have exerted sincere efforts to improve the papers quality by their constructive comments. Special thanks to the Field Chief Editor of Frontiers in Energy Research, Professor Uwe Schröder for giving the Guest Editorial Board this great chance.

\section{AUTHOR CONTRIBUTIONS}

The author confirms being the sole contributor of this work and has approved it for publication.

\section{ACKNOWLEDGMENTS}

I would also like to express my sincere gratitude and thanks to the team of Guest Editors (Professors Farhad Rachidi, Fei Gao, Hatem Zeineldin, Ke Meng, and Ehab El-Saadany) for their great efforts and time devoted to this Special Issue. Their valuable expertise and dedication have made it a reality.

Conflict of Interest: The author declares that the research was conducted in the absence of any commercial or financial relationships that could be construed as a potential conflict of interest.

Publisher's Note: All claims expressed in this article are solely those of the authors and do not necessarily represent those of their affiliated organizations, or those of the publisher, the editors and the reviewers. Any product that may be evaluated in this article, or claim that may be made by its manufacturer, is not guaranteed or endorsed by the publisher.

Copyright (C) 2021 Al-Durra. This is an open-access article distributed under the terms of the Creative Commons Attribution License (CC BY). The use, distribution or reproduction in other forums is permitted, provided the original author(s) and the copyright owner(s) are credited and that the original publication in this journal is cited, in accordance with accepted academic practice. No use, distribution or reproduction is permitted which does not comply with these terms. 